\title{
Cáncer pulmonar el peor efecto del tabaquismo
}

\author{
MARCIA ERAZO B.*, MAURICIO BUROTTO P.** y JUAN GUILLERMO GORMAZ A.***
}

\section{Lung cancer the worst effect of smoking}

Lung cancer is the first cancer death in Chile. Globally accounts for more than 8 million annual deaths, of the new cases of cancer, more than 6 million are smoking related, making smoking the most important cancer risk factor and cause of about $20 \%$ of the global cancer-related mortality. The incidence of lung cancer is directly related to smoking exposure level during life, exposure that increases the risk of this disease up to 30 times. This risk decreases exponentially with smoking cessation, especially if it occurs in early life stages. In Chile, $85 \%$ of lung cancers in men and $40 \%$ in women would be explained by smoking, equivalent to 1926 per year, being recommended to conduct brief counseling according to the Clinical Guidelines for the Treatment of Smoking in Chile 2017, and if it is not possible to refer all smokers with cancer to smoking cessation therapy that may be by telephone Salud Responde, MINSAL 6003607777, the number is in the cigarette packets.

Key words: Lung neoplasm; smoking cessation; smoking.

\section{Resumen}

El cáncer de pulmón constituye la primera causa de muerte por cáncer en Chile. A nivel mundial da cuenta de más de 8 millones de fallecimientos anuales, de los nuevos casos de cáncer, más de 6 millones están relacionados con tabaco, constituyendo a el tabaquismo un importante factor de riesgo y causa directa de cerca del $20 \%$ de la mortalidad global asociada a cáncer. La incidencia de cáncer de pulmón está directamente relacionada el nivel de exposición a tabaquismo durante la vida, la exposición puede aumentar el riesgo de esta enfermedad hasta 30 veces. El riesgo disminuye exponencialmente con la cesación de esta adicción, especialmente si ocurre en etapas tempranas de la vida. En Chile el $85 \%$ de los cánceres de pulmón en hombres y el $40 \%$ en mujeres estarían explicados por tabaquismo, equivalentes a 1926 anuales, siendo la recomendación realizar consejería breve según las Guias Clínicas de Tratamiento del Tabaquismo Chile 2017 y si no es posible derivar a todos los fumadores con cáncer a terapia de cesación del tabaquismo que puede ser telefónica Salud Responde MINSAL 6003607777, el número está en los envases de cigarrillos.

Palabras clave: Fumar; cáncer pulmonar; dejar de fumar.

Actualmente el Cáncer (CA) es la segunda causa de morbilidad y mortalidad a nivel mundial ${ }^{1}$. Según datos de la Organización Mundial de la Salud (OMS), el 2012 había 32,6 millones de pacientes con CA diagnosticado, 14 millones de nuevos casos y 8,2 millones de muertes, proyectándose un aumento del $70 \%$ para las próximas dos décadas ${ }^{2}$, lo que haría que se vuelva en pocos años en la primera causa de muerte. Excluyendo las neoplasias de piel no melanomas, el cáncer de pulmón ha sido el CA más prevalente por décadas y sigue en aumento. A nivel mundial da cuenta de más de 8 millones de fallecimientos anuales.

De los nuevos casos de cáncer general, más de 6 millones están relacionados con tabaquismo ${ }^{3}$ y de estos últimos dos tercios provienen de países

\footnotetext{
* Escuela de Salud Pública, Facultad de Medicina, Universidad de Chile.

** Jefe de Oncología, Clínica Alemana de Santiago.

*** Coordinador de Investigación en Oncología, Clínica Alemana de Santiago.
} 
menos desarrollados ${ }^{4}$, constituyendo al tabaquismo el más importante factor de riesgo de CA general y causa directa de cerca del $20 \%$ de la mortalidad global asociada a cáncer ${ }^{4,5}$. Específicamente el tabaquismo constituye factor de riesgo para a lo menos 19 tipos de $\mathrm{CA}^{6}$. Dentro de estos, esta adicción si sola daría cuenta del $70 \%$ de los CA de pulmón, bronquios y tráquea; del $42 \%$ de los CA orales, orofaríngeos y esofágicos; del $28 \%$ de los CA de vejiga y tracto urinario; del $22 \%$ de los CA de páncreas, del $14 \%$ de los CA de hígado; del 13\% de los CA de estómago; del 9\% de las Leucemias y del $2 \%$ de los CA cervicales 7 .

A nivel mundial, la incidencia de CA de pulmón está directamente relacionada con los niveles de exposición al humo de tabaco entre otras exposiciones $^{8,9}$, presentándose la menor incidencia en África Occidental $(1,7$ por cada 100.000$)$ y la mayor en Europa Central y del Este $(53,5 / 100.000)$ con mortalidades de 1,5/100.000 y 47,6/100.000 respectivamente. En relación con el género, la incidencia promedio en mujeres es más baja que la población general, oscilando entre $0,8 / 100.000$ en África Central y 33,8/100.000 en América del Norte, con mortalidades respectivas de $0,8 / 100.000$ y 23,5/100.000. El nivel de desarrollo económico-cultural también incide, dado que, si bien históricamente las más altas tasas de incidencia se han presentado en los países desarrollados, esta tendencia puede comenzar a revertirse ya que el 2012 los países menos desarrollados aportaron 1,1 millones sobre 1,8 millones de casos nuevos mundiales de CA de pulmón ${ }^{3}$.

La popularización del tabaquismo también influye en incidencias y mortalidades. En lugares donde el consumo de tabaco se popularizó primero, regiones más desarrolladas de América, Europa y Asia, las incidencias cáncer de pulmón han mostrado un retroceso a partir de la década de los 90 mientras que en los lugares donde el tabaquismo masivo empezó más tardíamente, regiones menos desarrolladas de los mismos continentes, ambos indicadores continúan en aumento. Desagregando por género se ha estimado que el $30 \%$ de las muertes por CA en hombres y el $10 \%$ en mujeres se deben específicamente a tabaquismo ${ }^{3}$, el cual es considerado el más prevenible de los factores de riesgo asociados a esta enfermedad ${ }^{4}$. En Chile, aún no se observa el efecto de la alta prevalencia de consumo de tabaco sobre la incidencia de cánceres, especialmente en mujeres, quienes han aumentado su consumo en los últimos años.

El tabaquismo aumenta el riesgo de cáncer pulmón entre 15 a 30 veces $^{10}$, incremento que está directamente relacionado con el nivel de exposición al humo de tabaco durante la vida. Sin embargo, este riesgo disminuye exponencialmente con la cesación del tabaquismo, especialmente si se produce en etapas tempranas de la vida ${ }^{11}$. En Chile se ha estimado que el $85 \%$ de los cánceres de pulmón en hombres y el $40 \%$ del de mujeres son explicados por consumo de tabaco, lo que implicaría que de la incidencia anual de cáncer de pulmón; 2.665 personas por año, 1926 estarían explicadas por el tabaco ${ }^{2}$, por lo que debe ser prioridad nacional erradicar su consumo.

Con relación a otros cánceres, de especial interés resulta la asociación entre tabaquismo y $\mathrm{CA}$ de colon y recto (CCR), que en su conjunto constituirían la cuarta causa de muerte por cáncer en Chile con mortalidades que hacia el 2010 se reportaron levemente superiores a 10/100.000 $0^{13}$. $\mathrm{Si}$ bien estas tasas son inferiores a las de países occidentales post-transición epidemiológica con elevados niveles de tabaquismo como Estados Unidos, con tasas de 14,45/100.000 el $2013^{14}$, en Chile la mortalidad por estos cánceres casi se duplica en una década ${ }^{3}$. La evidencia internacional ha reportado que el tabaco aumenta el riesgo de estos cánceres entre dos a tres veces ${ }^{15}$, dado que la elevada irrigación de la mucosa colorrectal favorece la exposición de estos tejidos a cancerígenos provenientes del tabaco.

En pacientes con CA que siguen fumando $\mathrm{y}$ más aún en pacientes con $\mathrm{CA}$ asociados a tabaquismo que fuman, existe un aumento considerable del riesgo de empeorar su enfermedad y de desarrollar nuevas enfermedades. Esta población es de especial importancia para la cesación del tabaquismo, dado el rol del tabaco en la potenciación del cáncer y comorbilidades asociadas $^{16}$, por lo que la recomendación es realizar ABC-D según las Guías Clínicas de Tratamiento del Tabaquismo Chile 2017 y si no es posible derivar a todos los fumadores con cáncer a terapia de cesación del tabaquismo que puede ser telefónica, Salud Responde MINSAL 6003607777, el número está en los envases de cigarrillos.

\section{Bibliografía}

1.- ERAZO M, NES K. Cigarette Smoking: Health Effects and Challenges for Tobacco Control. Editorial NOVA, Nueva York, Estados Unidos, 2017.

2.- STEWART B, WILD C. (eds.), International Agency for Research on Cancer, WHO. World Cancer Report 2014 (Online). Disponible en:: http://www.iarc.fr/en/ publications/books/wcr/wcr-order.php (Consultado el 9 de mayo de 2016).

3.- FERLAY J, SOERJOMATARAM I, DIKSHIT R, 
ESER S, MATHERS C, REBELO PARKIN D, et al. Cancer Incidence and Mortality Worldwide: Sources, Methods and Major Patterns in GLOBOCAN 2012. Int J Cancer. 2015; 136: E359-86.

4.- LEE Y, HASHIBE M. Tobacco, alcohol, and cancer in low and high income countries. Ann Glob Health. 2014; 80: 378-83.

5.- LIM S, VOS T, FLAXMAN A, DANAEI G, SHIBUYA K, ADAIR-ROHANI H, et al. A Comparative Risk Assessment of Burden of Disease and Injury Attributable to 67 Risk Factors and Risk Factor Clusters in 21 Regions, 1990-2010: A Systematic Analysis for the Global Burden of Disease Study 2010. Lancet. 2012; 380: 2224-60.

6.- SCHOTTENFELD D, BEEBE-DIMMER J, BUFFLER P, OMENN G. Current perspective on the global and United States cancer burden attributable to lifestyle and environmental risk factors. Annu Rev Public Health. 2013; 34: 97e117.

7.- GORMAZ J, CORTEZ I, HENRÍQUEZ P, SOTOMAYOR C, GAJARDO A, NES K, et al. Capítulo: Tobacco and Cancer; En libro: Cigarette smoking: Health effects and challenges for tobacco control. Editorial NOVA, Nueva York, Estados Unidos, 2017.

8.- EZZATI M, HENLEY S, LÓPEZ A, THUN M. Role of smoking in global and regional cancer epidemiology: current patterns and data needs. Int J Cancer. 2005; 116 : 963-71.
9.- THUN M, PETO R, BOREHAM J, LÓPEZ A. Stages of the cigarette epidemic on entering its second century. Tob Control. 2012; 21: 96-101.

10.- VINEIS P, ALAVANJA M, BUFFLER P, FONTHAM E, FRANCESCHI S, GAO Y, et al. Tobacco and cancer: recent epidemiological evidence. J Natl Cancer Inst. 2004; 96: 99e106.

11.- WARREN G, CUMMINGS K. Tobacco and lung cancer: risks, trends, and outcomes in patients with cancer. Am Soc Clin Oncol Educ Book. 2013: 359-64.

12.- ERAZO M, AMIGO H, OYARZÚN M, PERUGA A. Tabaquismo activo y cáncer pulmonar: Determinación de fracciones atribuibles por sexo. Rev Med Chile 2008; 136: 1272-80.

13.- ROCO A, QUIÑONES L, ACEVEDO C, ZAGMUTT O. Situación del cáncer en Chile 2000-2010. Cuad Méd Soc (Chile). 2013; 53: 83-94.

14.- MARLEY A, NAN H. Epidemiology of colorectal cancer. Int J Mol Epidemiol Genet. 2016; 7: 105-14.

15.- BOTTERI E, IODICE S, BAGNARDI V, RAIMONDI S, LOWENFELS AB, MAISONNEUVE P. Smoking and colorectal cancer: a meta-analysis. JAMA. 2008; 300: 2765-78.

16.- Clinical Practice Guideline. Rockville, MD: U.S. Department of Health and Human Services. Public Health Service, May 2008. Disponible en: http://www.ncbi. nlm.nih.gov/books/NBK63952/ (Consultado el 9 de mayo de 2016).

Correspondencia a:

B.Q. Juan Guillermo Gormaz A.

Email: jgormaz@alemana.cl 